\title{
Comparing Outcomes for Rare Primary Hepatic Tumors after Liver Transplantation
}

\author{
Nhu Thao T Nguyen ${ }^{1 *}$, Theresa R Harring ${ }^{1}$, Jacfranz J Guiteau¹, Ron T Cotton ${ }^{1}$, Ismael Salas de Armas ${ }^{1}$, Hao Liu ${ }^{2}$, John A Goss ${ }^{1}$ and \\ Christine A O'Mahony ${ }^{1}$
}

${ }^{1}$ Division of Abdominal Transplantation and Hepatobiliary Surgery, Michael E. DeBakey Department of Surgery, Baylor College of Medicine, 1709 Dryden Road Suite 1500, Houston, Texas 77030

${ }^{2}$ Division of Biostatistics, Dan L. Duncan Cancer Center, Department of Medicine, Section of Hematology/Oncology, Baylor College of Medicine, One Baylor Plaza, 450A, Houston, Texas 77030

\begin{abstract}
Aim: Liver transplantations (LT) have proven to be a successful treatment for many tumors of the liver. The goal of this study was to evaluate the outcomes of liver transplantations in patients with primary liver tumors with a focus on rarer malignancies.

Methods: The UNOS database catalogues all adult patients who underwent LT for a primary liver tumor from 1992-2008. Of the 73,231 liver transplantations, 5,682 patients with liver tumors were identified and categorized by indication for LT: hepatocellular carcinoma (HCC, $n=5272)$, hepatic epithelioid hemangioendothelioma $(\mathrm{HEH}$, $n=85)$, cholangiocarcinoma $(n=249)$, sarcomas $(n=11)$ and combined HCC-Cholangiocarcinomas (HCC-CC, $n=12)$. Survivals were calculated using Kaplan-Meier and log rank tests.

Results: 5,629 patients received LT for solid liver tumors. HCC patients and their allografts survived longer than those transplanted for cholangiocarcinomas $(p=0.001,0.002)$ or for HCC-CC $(p=0.025,0.004)$. Overall survival rates of HCC patients were $86.4 \%, 71.3 \%$, and $61.2 \%$ at 1,3 and 5 years, respectively. Cholangiocarcinoma patients had survival rates of $79.7 \%, 60.3 \%$ and $45.5 \%$ at 1 -, 3 - and 5 -years from transplant. HCC-CC patients had the worst overall survival of $72.9 \%, 39.1 \%$ and $39.1 \%$ at 1,3 and 5 years. Allograft survival in HCC-CC patients was comparatively low, averaging $65.6 \%, 35.2 \%$ and $0 \%$ at $1-, 3$ - and 5 -years. $\mathrm{HEH}$ patients and their allografts survived the longest with overall survival of $83.9 \%, 77.8 \%$ and $73.5 \%$ at 1,3 and 5 years, respectively, and allograft survival at $76.8 \%, 69.8 \%$ and $64.3 \%$.

Conclusion: Our data reveals overall survival was significantly better in HEH patients when compared to HCC, cholangiocarcinoma and HCC-CC patients after LT. In fact, HCC-CC patients fared the worst, both in patient and allograft survival, as compared to $\mathrm{HCC}$ and $\mathrm{HEH}$. Results of cholangiocarcinoma patients show worse survival after LT compared to HCC and HEH, though recent evidence suggests adjuvant therapy will change outcomes for the future. Our findings suggest transplantation for HCC-CC may not be sufficient treatment. Other forms of adjuvant and neoadjuvant therapy may be indicated, warranting further research.
\end{abstract}

Keywords: Liver transplantation; Primary liver malignancy; Survival analysis; Combined hepatocellular carcinoma-cholangiocarcinoma; Hepatic epithelioid hemangioendothelioma; Soft tissue sarcoma

Abbreviations: UNOS: United Network for Organ Sharing; LT: Liver Transplantation; OLT: Orthotopic Liver Transplantation; MELD: Model for End-Stage Liver Disease; HCC: Hepatocellular Carcinoma; HB: Hepatoblastoma, HEH: Hepatic Epithelioid Hemangioendothelioma, NET: Neuroendocrine tumors; HCC- CC: Combined Hepatocellular Carcinoma and Cholangiocarcinoma; Lrx: Liver resection

\section{Introduction}

Liver transplantation (LT) serves as a critical treatment modality for end-stage liver disease and a certain subset of hepatic tumors. For hepatic tumors, its role has evolved to include neoadjuvant chemotherapy and radiotherapy for many primary liver cancers with improved outcomes over the years [1]. LT has proven to be a well-established and successful treatment for hepatocellular carcinoma (HCC), the predominant primary cancer of the liver worldwide [2-4]. Cholangiocarcinoma is another primary liver tumor, second greatest in frequency though with much poorer long-term prognosis $[1,5,6]$. The outcomes of these patients after LT are well documented; [6-9] however, less data exists in regards to outcomes of transplantations for rarer malignancies of the liver, such as hepatic epithelioid hemangioendothelioma (HEH) $[10,11]$ or combined hepatocellular carcinoma-cholangiocarcinomas (HCC-CC) [12]. Frequencies of these tumors vary greatly according to world region. Little data on these malignancies come from North America, where the incidence of these rarer tumors is lower. The infrequency of these tumors often makes single-center experiences limited in both scope and power of study. The aim of this study is to further characterize the outcomes of liver transplantations performed for primary liver tumors on a national level.

Primary liver tumors comprise $4 \%$ of cancer diagnoses globally. HCC is diagnosed in 4.8 per 100,000 people annually, whereas sarcomas and $\mathrm{HEH}$ are found in $<0.1$ per 100,000 people in the general population [13]. Cholangiocarcinoma occurs in 2.5 per 100,000 people. Combined HCC-CC has been reported to occur in as few as $1 \%$ to as many as $14.3 \%$ of the population depending on locale [14,15]. It becomes more evident how such infrequent tumors with varying degrees of predictability in clinical course can be difficult to characterize and

*Corresponding author: Nhu Thao T. Nguyen, Michael E. DeBakey Department of Surgery, 1709 Dryden, Ste 1537, Houston, TX 77030, Tel. 713.798.7949; Fax: 713.798.8319; E-mail: tnnguyen@bcm.edu

Received November 01, 2011; Accepted December 09, 2011; Published December 13, 2011

Citation: Nguyen NTT, Harring TR, Guiteau JJ, Cotton RT, de Armas IS, et al (2011) Comparing Outcomes for Rare Primary Hepatic Tumors after Liver Transplantation. J Transplant Technol Res 1:106. doi:10.4172/2161-0991.1000106

Copyright: (c) 2011 Nguyen NTT, et al. This is an open-access article distributed under the terms of the Creative Commons Attribution License, which permits unrestricted use, distribution, and reproduction in any medium, provided the original author and source are credited. 
treat with a standardized therapeutic approach $[1,11]$. Currently, LT yields excellent results for patients with HCC and remains a standard indication for OLT in selected patients $[13,16]$. Results for HEH are increasingly reported to be satisfactory as well for cases detected earlier in the clinical course with adjuvant therapy, though has yet to be proven as a durable treatment from lack of larger case series $[1,10,11,13,17,18]$. HCC-CC, on the other hand, offers poorer prognosis in recent review $[19,20]$. This study compares and contrasts the outcomes of patients with HCC, HEH, cholangiocarcinoma, sarcomas and combined HCCCC who underwent a LT in the United States in the past 16 years to gain a better general understanding of our progress in the field of primary liver tumors and the role of LT as treatment.

\section{Methods}

LT cases were reviewed in the retrospectively maintained and comprehensive United Network Organ Sharing/Organ Procurement Transplantation Network (UNOS/OPTN) dataset which catalogues all transplantations in the United States to compare and contrast the survival experiences between the tumor types. The UNOS/OPTN database was used to select for all 73,231 adult ( $\geq 18$ years old) patients who underwent LT between 1992-2008. Of those, patients with a primary liver tumor were identified in 5,629 cases. These 5,629 patients were divided into pre-operative indications for OLT and the categories of tumor types used for comparisons were hepatocellular carcinoma (HCC, $\mathrm{n}=5272$ ), hepatic epithelioid hemangioendothelioma ( $\mathrm{HEH}$, $\mathrm{n}=85)$, cholangiocarcinoma $(\mathrm{n}=249)$, sarcomas $(\mathrm{n}=11)$ and combined HCC-CC ( $\mathrm{n}=12)$. Exclusion criteria included Status-1 patients, multiorgan recipients and retransplanted patients.

Time-to-event data were obtained from this database to estimate post-OLT survival. Specifically, the time variable was calculated as the length of time between transplantation and either death or last known follow-up. An observation was censored if the individual was alive at the last known follow-up. Patient and allograft survival rates were determined using the Kaplan-Meier test and the log-rank test. These groups and their overall patient and allograft survival experiences were compared to each other with log-rank pairwise comparisons to evaluate for difference, with a p-value $\leq 0.05$ considered statistically significant. This total sample was then divided into two groups: patients transplanted for combined HCC-CC and patients transplanted for all other primary liver tumor types to determine any significance between these two groups. SPSS version 15.0 (SPSS Inc., IBM Corporation, Chicago, IL, USA) was used for all statistical analyses.

\section{Results}

Of the cases reviewed, there was a male predominance of $77.4 \%$. The patients were primarily Caucasian, accounting for $68.4 \%$ of the group, while Hispanics made up the larger minority at $13.7 \%$. The median age of recipients was 55.48 years. The mean wait time on the transplant list was 235 days. The majority of patients received whole liver allografts $(95.0 \%)$, compared to split liver allografts. The mean length of postoperative stay after LT was 13 days. Median follow-up time within this database was 8.4 years \pm 64 days.

Patient survival rates of HCC patients were $86.4 \%, 71.3 \%$, and $61.2 \%$ at 1-, 3- and 5-years, respectively, with allograft survival similarly trending at $82.7 \%, 67.3 \%$ and $57.5 \%$, respectively (Tables 1 and 3). Cholangiocarcinoma patients had survival rates of $79.7 \%$, $60.3 \%$ and $45.5 \%$ at 1-, 3 - and 5-years from transplant, with allografts surviving $76.3 \%, 57.2 \%$ and $43.2 \%$, respectively. Patients transplanted for HCC and their allografts survived significantly longer than those transplanted for cholangiocarcinomas ( $\mathrm{p}=0.001, \mathrm{p}=0.002$, respectively) or for combined HCC-CC ( $p=0.025, p=0.004$, respectively). Survival of patients with cholangiocarcinoma after LT is statistically worse than HCC and HEH patients $(p=0.001, p=0.001)$. The patient survival comparisons between tumor types are visualized in Figure 1.

HEH patients and their allografts survived the longest of all liver tumors with overall survival of $83.9 \%, 77.8 \%$ and $73.5 \%$ at 1,3 and 5 years, respectively, and graft survival at $76.8 \%, 69.8 \%$ and $64.3 \%$. This

\begin{tabular}{|l|l|l|l|}
\hline Solid Tumor Types & 1-Year Survival (Std. Error) & 3-Year Survival (Std. Error) & 5-Year Survival (Std. Error) \\
\hline Hepatocellular Carcinoma (5272) & $86.4(0.5)$ & $71.3(0.8)$ & 61.2 (1.1) \\
\hline Hepatic Epithelioid Hemangioendothelioma (85) & $83.9(4.3)$ & $77.7(5.0)$ & $73.5(5.5)$ \\
\hline Cholangiocarcinoma (249) & $79.7(2.8)$ & $60.3(3.9)$ & $45.5(4.8)$ \\
\hline Sarcoma (11) & $78.8(13.4)$ & $45.0(16.6)$ & $45.0(16.6)$ \\
\hline Combined HCC-CC(12) & $72.9(13.5)$ & $39.1(16.3)$ \\
\hline
\end{tabular}

Table 1: Overall Patient Survival Time between Primary Liver Tumor Types.

Log Rank Pairwise Comparisons of Patient Survival between Solid Tumor Types

\begin{tabular}{|c|c|c|c|c|c|}
\hline Solid Tumor Types & Hepatocellular Carcinoma & Hepatic Epithelioid Hemangioendothelioma & Cholangiocarcinoma & Sarcoma & Combined HCC-CC \\
\hline & Significance $(p \leq 0.05)$ & & & & \\
\hline Hepatocelluar Carcinoma & & .017 & .001 & .993 & .025 \\
\hline $\begin{array}{l}\text { Hepatic Epithelioid } \\
\text { Hemangioendothelioma }\end{array}$ & .017 & & .001 & .245 & .016 \\
\hline Cholangiocarcinoma & .001 & .001 & & .758 & .321 \\
\hline Sarcoma & .993 & .245 & .758 & & .843 \\
\hline Combined HCC-CC & .025 & .016 & .321 & .843 & \\
\hline
\end{tabular}

Table 2: Pairwise Comparisons of Patient Survivals between Primary Liver Tumor Types. ( $p$-values of significance, ie. $\leq 0.05$, are shaded gray).

\begin{tabular}{|l|l|l|l|}
\hline Solid Tumor Types & 1-Year Survival (Std. Error) & 3-Year Survival (Std. Error) & 5-Year Survival (Std. Error) \\
\hline Hepatocellular Carcinoma (5272) & $82.7(0.6)$ & $67.3(0.8)$ & $57.5(1.0)$ \\
\hline Hepatic Epithelioid Hemangioendothelioma (85) & $76.8(4.7)$ & $69.8(5.2)$ & $64.3(5.7)$ \\
\hline Cholangiocarcinoma (249) & $76.3(2.9)$ & $57.2(3.8)$ & $43.2(4.6)$ \\
\hline Sarcoma (11) & $71.6(1.4)$ & $40.9(15.6)$ & $40.9(15.6)$ \\
\hline Combined HCC-CC (12) & $65.6(14.0)$ & $35.2(15.2)$ \\
\hline
\end{tabular}

Table 3: Allograft Survival Time between Primary Liver Tumor Types. 
trend is statistically significant when comparing overall patient survival to all other tumor types except hepatic sarcomas. Sarcomas trended below HCC, HEH and cholangiocarcinomas, but never reached significance when compared to any other tumor type. Sarcoma patients had patient survivals of $78.8 \%, 45.0 \%$ and $45.0 \%$ and allograft survivals of $71.6 \%, 40.9 \%$ and $40.9 \%$ at 1 -, 3- and 5-years, respectively.

HCC-CC patients had the worst overall survival of $72.9 \%, 39.1 \%$ and $39.1 \%$ at 1,3 and 5 years, significantly worse when compared to HCC and HEH patients. Furthermore, graft survival in HCC-CC patients was comparatively low, at $65.6 \%, 35.2 \%$ and $0 \%$ at 1 -, 3 - and 5 -years. Tables 2 and 4 illustrate the significance of overall patient and allograft survival.

Patient survival experience did not become dramatically different between the 5 groups until after the first year after transplantation. HCC patients had the best patient survival in the first year, with a survival experience significantly higher than that of cholangiocarcinoma and combined HCC-CC patients (Table 2); HEH patients had better longterm survivals than HCC by 5 years post-transplantation $(\mathrm{p}=0.017)$ though this difference was not significant when comparing allograft survival of the two patient groups (NS, $\mathrm{p}=0.104$ ).

To further characterize the outcome of HCC-CC patients, we then compared this group to all other LT patients who received an allograft for a solid tumor type. In this comparison, HCC-CC patients were found to do significantly worse than all other solid tumor types and their patients, both in regards to overall patient survival, and also in terms of allograft survival (Figure 3$)(p=0.029, p=0.005)$ reaffirming the poor outcome of this patient subgroup when compared to patients with other tumor types (Table 5).

\section{Discussion}

Outcomes after LT have improved with advances in immunosuppression, perioperative care, surgical technique and patient selection. Characterizing outcomes helps to reflect on the current system and find opportunities to improve. HCC is the most common primary tumor of the liver and the most common tumor to be transplanted $[3,21]$. Currently, HCC survival after LT at 5-years approximates $55-70 \%$, significantly improving with the introduction

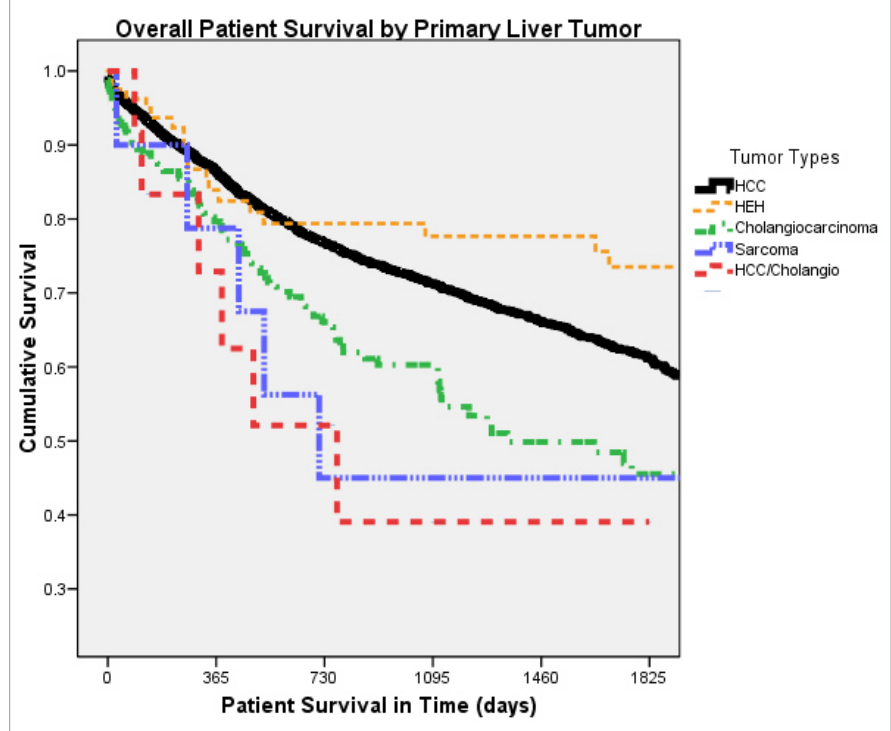

Figure 1: Overall Patient Survival by Primary Liver Tumor Type.

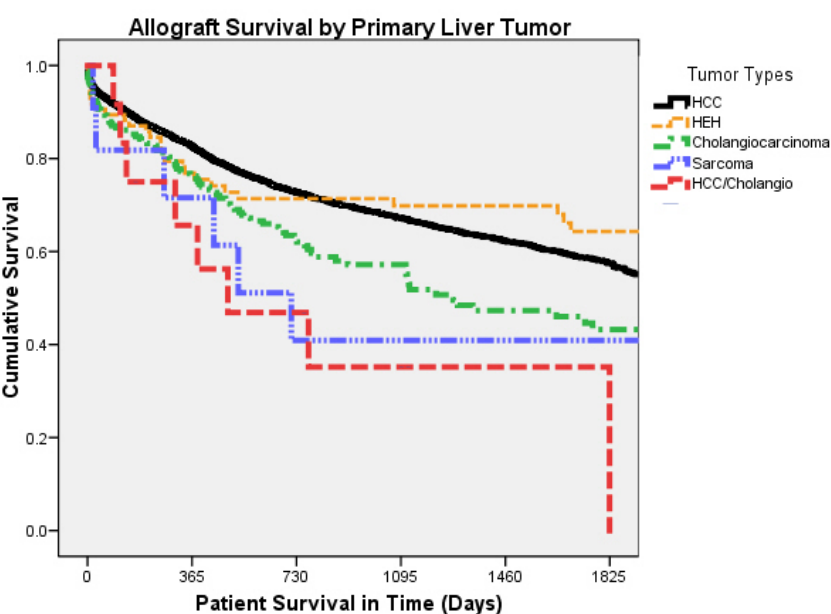

Figure 2: Overall Allograft Survival by Primary Liver Tumor Type.

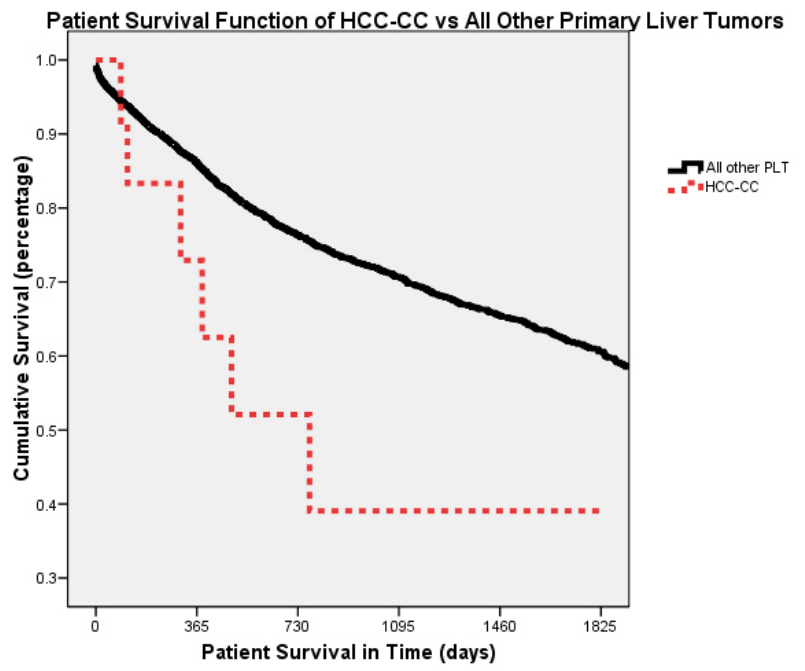

Figure 3: Overall Patient Survival between Combined HCC-CC and all other primary liver tumors after OLT.

of Milan criteria to organ allocation in 2002 [22-24]. While this study focuses on rare primary malignancies of the liver, the results are compared to the outcomes of HCC patients after LT as the most commonly transplanted malignancy of the liver, accounting for a quarter of all liver transplantations in the US [24].

When comparing the survival experiences of primary liver tumor patients treated with LT in the past 16 years, the data reveals overall survival was significantly better in $\mathrm{HEH}$ patients when compared to HCC, cholangiocarcinoma and combined HCC-CC patients.

A review of the recent literature on primary malignant $\mathrm{HEH}$ by Mehrabi et al. collected information on 286 patients for whom treatment data was reported, and presented survival outcomes stratified by clinical or surgical management. Liver resection patients collected in this review had survivals of $100 \%$ and $75 \%$ at 1 - and 5 -years. Patients who had no treatment had calculated survivals at 1 - and 5 -years of $39.3 \%$ and $4.5 \%$, respectively, compared to those with transplanted livers, who had $96 \%$ and $54.5 \%$ survivals at 1 - and 5 -years [11]. The data presented here does not reflect such a high 1-year survival for $\mathrm{HEH}$ patients after 
Log Rank Pairwise Comparisons of Allograft Survival between Solid Tumor Types

\begin{tabular}{|c|c|c|c|c|c|}
\hline Solid Tumor Types & Hepatocellular Carcinoma & Hepatic Epithelioid Hemangioendothelioma & Cholangiocarcinoma & Sarcoma & Combined HCC-CC \\
\hline & \multicolumn{5}{|l|}{ Significance $(p \leq 0.05)$} \\
\hline Hepatocelluar Carcinoma & & .104 & .002 & .898 & .004 \\
\hline $\begin{array}{l}\text { Hepatic Epithelioid } \\
\text { Hemangioendothelioma }\end{array}$ & .104 & & .015 & .353 & .014 \\
\hline Cholangiocarcinoma & .002 & .015 & & .739 & .109 \\
\hline Sarcoma & .898 & .353 & .739 & & .569 \\
\hline Combined HCC-CC & .004 & .014 & .109 & .569 & \\
\hline
\end{tabular}

Table 4: Pairwise Comparisons of Allograft Survivals between Primary Liver Tumor Types. ( $p$-values of significance, ie. $\leq 0.05$, are shaded gray).

\begin{tabular}{|l|l|l|l|}
\hline Patient Survival & 1-Year Survival (std. error) & 3-Year Survival (std. error) \\
\hline Other Primary Liver Tumors (5617) & $86.1(0.05)$ & $70.7(0.08)$ & 5-Year Survival (std. error) \\
\hline Combined HCC-CC (12) & $72.9(13.5)$ & $39.1(16.3)$ & $60.6(0.1)$ \\
\hline Allograft Survival & & $66.6(0.8)$ & $39.1(16.3)$ \\
\hline Other Primary Liver Tumors (5617) & $62.3(0.05)$ & $35.2(15.2)$ & $56.8(0.1)$ \\
\hline Combined HCC-CC (12) & $65.6(14.0)$ & 0 \\
\hline
\end{tabular}

Table 5: Patient and allograft survival outcomes between Combined HCC-CC and all other primary liver tumor types. ( $p=0.029, p=0.005$, respectively).

LT, but the 5-year survival is markedly improved. Rodriguez at al. also looked at the UNOS/OPTN database for HEH alone, and found the same 1- and 5-year survivals from 1987-2005 [10]. Comparatively, a study of the European Liver Transplant Registry analyzed 59 patients and their survival outcomes after LT, $93 \%$ and $83 \%$ at 1 - and 5 -years post-transplant, finding nodal invasion and extrahepatic disease did not significantly affect outcome [25]. A study of the Mayo Clinic retrospectively reviewed 30 patients with $\mathrm{HEH}$ who underwent liver resection, LT, chemotherapy or no therapy and found liver resection to be a comparable option for surgical treatment compared to LT when allowing for patient selection [26]. Similar to Mehrabi et al.'s review, liver resection (Lrx) candidates had better survival outcomes; this is possibly attributed to a lower stage at presentation. Mehrabi et al.'s group reported 27 patients who had Lrx, only $9.4 \%$ of the sample size compared to $44.8 \%$ who underwent LT. Lrx patients are likely healthier with less of a tumor burden at presentation and presumably, a better prognosis. Until further prospective, multi-center trials are performed, LT remains a viable treatment option for a large portion of $\mathrm{HEH}$ patients, with excellent outcomes reported in this study.

Cholangiocarcinoma patients transplanted in the past 16 years had poorer outcomes, significantly so when compared to the cohort with HCC and HEH. A recent Cochrane review of the literature reports a $39 \%$ pooled survival rate by 5 -years, concordant with this study's findings [27]. Recently, cholangiocarcinoma treatment has evolved to include neoadjuvant chemoradiation therapy, yielding a 5-year survival rate of $73 \%$ from a prospective cohort of 120 patients [6]. Another smaller series of cholangiocarcinoma patients performed by Punjala et al. studied the effects of neoadjuvant chemoradiation therapy prior to LT on a cohort of 22 patients and found significant improvement in projected survival [27]. Gu et al.'s meta-analysis found the reports integrating preoperative adjuvant therapies had improved (survival at 5 -years to $65 \%$ ). As the future brings improved adjuvant therapies, undoubtedly predicted survival rates for this disease will increase over time.

Hepatic sarcomas of the liver are not often transplanted in the US, as reflected by the small sample size in this study. The predicted survivals found in this report show a trend similarly poor compared to cholangiocarcinoma patients, though without statistical significance, it is difficult to draw conclusions. Both primary and metastatic sarcomas of the liver have, in limited series and of varying histologies, proven to have prohibitive outcomes in general. A small prospective study done in Germany did find a 65\% survival rate at 5 -years in their cohort of 22 patients, finding histology was a significant factor in prognosis [28]. Currently, however, hepatic sarcoma is not a diagnosis eligible for transplantation in the US, in large part due to the dismal outcomes reflected in this study and in recent literature [29-31].

Combined HCC-CC patients fared the worst when compared to all the primary liver tumors studied, significantly so when compared to HCC and HEH. Other studies have shown HCC-CC also fare worse when compared to cholangiocarcinoma alone, though this analysis did not show this trend to be statistically significant $[1,5,6,32]$. When this subgroup was compared to all LTs performed for the indication of a primary liver tumor, HCC-CC continued to have a dismal prognosis, significantly so in terms of overall patient and allograft survival.

HCC-CC is a rare malignancy comprised of histopathological features of both hepatocellular carcinoma and cholangiocarcinoma [33]. Preoperative diagnosis is difficult, and in fact, most patients are misdiagnosed as having HCC or cholangiocarcinoma [14,34]. In the UNOS/OPTN database, diagnosis entered is made preoperatively and is the indication for listing and transplantation. Index of suspicion for HCC-CC may be raised with increased CA 19-9, Alpha-fetoprotein serum levels and imaging studies, prompting biopsies and ductal brushings, though often pathological diagnosis is confirmed after LT $[15,34]$. Hepatic resection with hilar lymph node dissection is the surgical treatment of choice when preoperative diagnosis is made. An Italian study in 2008 found 18 patients with combined HCC-CC who had disease-specific survival to be $90.9 \%, 62.3 \%$ and $62.3 \%$ at $1-, 3-$ and 5-year survival after hepatic resection, with hilar nodal resection for 8 of those patients [12]. The role of LT for HCC-CC, on the other hand, is still controversial, with data lacking of outcomes and results $[15,35,36]$. One study analyzed the SEER registry to find whether cancer-directed therapies changed survival outcomes. Of 380 patients with HCC-CC, 20 received LT as their cancer-directed therapy and analysis proved transplantation did indeed correlate with improved outcomes compared to those who did not receive cancer-directed treatment [20]. Here, we are able to identify a dozen cases documented to have undergone liver transplantation with the diagnosis of HCC-CC in the United States with long-term follow-up. For this analysis, LT for HCC-CC does not offer hopeful prospects relative to all other primary tumors of the liver, and warrants further investigation.

In using the UNOS/OPTN dataset to assess outcomes, there are limitations which deserve acknowledgement. Regrettably, this database does not contain certain specific clinical data, including location of 
primary liver tumors which has implications for management and outcomes. Tumor stage at initial presentation is unknown, as well as final pathological staging and time of diagnosis, which may underrepresent the actual number of cancer cases present in the transplant population. Treatment, preoperatively and postoperatively, are not recorded, and neither are tumor markers and histological signs which have been shown to influence recurrence and survival, including CA 19-9, vascular invasion, tumor size, location and multiplicity $[14,15]$. This makes comparisons between stages and standardization of care challenging.

However, the survival curves we report here represent overall outcomes of primary liver tumors which continue to be important, both in patient education, organ allocation and post-transplant care. This data illustrates outcomes of current transplant management for primary liver tumors. Rather than deriving from a single institution's experience, information is gathered on outcomes of a national experience, increasing the sample size and power of the results, which on rare diseases would be otherwise difficult to describe. The values reported here serve as salient pieces of data to the informed transplant team and transplant patient, as part of their clinical care and decisionmaking.

Although survival outcomes after transplantation with more common tumors have been well established, our findings suggest that patients undergoing LT for HEH also carry acceptable outcomes, but that for patients with combined HCC-CC, LT may not be an ideal treatment alone. Other forms of adjuvant and neoadjuvant therapy may be indicated for these patients. Patients with cholangiocarcinoma have poorer outcomes after LT, though recent changes in adjuvant therapy and medical management may improve survival.

\section{References}

1. Grossman EJ, Millis JM (2010) Liver transplantation for non-hepatocellular carcinoma malignancy: Indications, limitations, and analysis of the current literature. Liver Transpl 16: 930-942.

2. El-Serag HB (2007) Epidemiology of hepatocellular carcinoma in USA. Hepatol Res 37 Suppl 2: S88-S94.

3. Nordenstedt H, White DL, El-Serag HB (2010) The changing pattern of epidemiology in hepatocellular carcinoma. Dig Liver Dis 42: S206-S214.

4. Rahbari NN, Mehrabi A, Mollberg NM, Müller SA, Koch M, et al. (2011) Hepatocellular Carcinoma: current management and perspectives for the future. Ann Surg 253: 453-469.

5. Lee JH, Chung GE, Yu SJ, Hwang SY, Kim JS, et al. (2011) Long-term prognosis of combined hepatocellular and cholangiocarcinoma after curative resection comparison with hepatocellular carcinoma and cholangiocarcinoma. J Clin Gastroenterol 45: 69-75.

6. Rosen CB, Heimbach JK, Gores GJ (2010) Liver transplantation for cholangiocarcinoma. Transpl Int 23: 692-697.

7. Hong JC, Jones CM, Duffy JP, Petrowsky H, Farmer DG, et al. (2011) Comparative analysis of resection and liver transplantation for intrahepatic and hilar cholangiocarcinoma: a 24-year experience in a single center. Arch Surg 146: 683-689.

8. Ho CM, Lee PH, Chen CL, Ho MC, Wu YM, et al. (2011) Long-Term Outcomes After Resection Versus Transplantation for Hepatocellular Carcinoma Within UCSF Criteria. Ann Surg Oncol.

9. Hanish SI, Knechtle SJ (2011) Liver transplantation for the treatment of hepatocellular carcinoma. Oncology (Williston Park) 25: 752-757.

10. Rodriguez JA, Becker NS, O'Mahony CA, Goss JA, Aloia TA (2007) Long-Term Outcomes Following Liver Transplantation for Hepatic Hemangioendothelioma: The UNOS Experience from 1987 to 2005. J Gastrointest Surg 12: 110-116.

11. Mehrabi A, Kashfi A, Fonouni H, Schemmer P, Schmied BM, et al. (2006) Primary malignant hepatic epithelioid hemangioendothelioma: a comprehensive review of the literature with emphasis on the surgical therapy. Cancer 107: 2108-2121.
12. Portolani N, Baiocchi GL, Coniglio A, Piardi T, Grazioli L, et al. (2008) Intrahepatic Cholangiocarcinoma and Combined Hepatocellular-Cholangiocarcinoma: A Western Experience. Ann Surg Oncol 15: 1880-1890.

13. Hertl M, Cosimi AB (2005) Liver transplantation for malignancy. Oncologist 10 : 269-281.

14. Kim KH, Lee SG, Park EH, Hwang S, Ahn CS, et al. (2009) Surgical Treatments and Prognoses of Patients with Combined Hepatocellular Carcinoma and Cholangiocarcinoma. Ann Surg Oncol 16: 623-629.

15. Kassahun WT, Hauss J (2008) Management of combined hepatocellular and cholangiocarcinoma. Int J Clin Pract 62: 1271-1278.

16. Zarrinpar A, Kaldas F, Busuttil RW (2011) Liver transplantation for hepatocellular carcinoma: an update. Hepatobiliary Pancreat Dis Int 10: 234-242.

17. Guiteau JJ, Cotton RT, Karpen SJ, O’Mahony CA, Goss JA (2010) Pediatric liver transplantation for primary malignant liver tumors with a focus on hepatic epithelioid hemangioendothelioma: The UNOS experience. Pediatr Transplant 14: $326-331$

18. Bonaccorsi-Riani E, Lerut JP (2010) Liver transplantation and vascular tumours. Transplant Int 23: 686-691.

19. Panjala C, Senecal DL, Bridges MD, Kim GP, Nakhleh RE, et al. (2010) The diagnostic conundrum and liver transplantation outcome for combined hepatocellular-cholangiocarcinoma. Am J Transplant 10: 1263-1267.

20. Wang J, Wang F, Kessinger A (2010) Outcome of Combined Hepatocellular and Cholangiocarcinoma of the Liver. J Oncol 2010: 1-7.

21. Stewart BW, Kleihues $P$ (2003) International Agency for Research on Cancer. World cancer report. Lyon: IARC Press.

22. Gordon-Weeks AN, Snaith A, Petrinic T, Friend PJ, Burls A, et al. (2011) Systematic review of outcome of downstaging hepatocellular cancer before liver transplantation in patients outside the Milan criteria. Br J Surg 98: 1201 1208

23. Hoffmann K, Hinz U, Hillebrand N, Radeleff BA, Ganten TM, et al. (2011) Risk factors of survival after liver transplantation for HCC: a multivariate singlecenter analysis. Clin Transplant 25: E541-E551.

24. Samuel D, Colombo M, El-Serag H, Sobesky R, Heaton N (2011) Toward optimizing the indications for orthotopic liver transplantation in hepatocellular carcinoma. Liver Transpl 17: S6-S13.

25. Lerut JP, Orlando G, Adam R, Schiavo M, Klempnauer J, et al. (2007) The place of liver transplantation in the treatment of hepatic epitheloid hemangioendothelioma: report of the European liver transplant registry. Ann Surg 246: 949-957.

26. Grotz TE, Nagorney D, Donohue J, Que F, Kendrick M, et al. (2010) Hepatic epithelioid haemangioendothelioma: is transplantation the only treatment option? HPB (Oxford) 12: 546-553.

27. Panjala C, Nguyen JH, Al-Hajjaj AN, Rosser BA, Nakhleh RE, et al. (2011) The impact of neoadjuvant chemoradiation on the tumor burden prior to live transplantation in unresectable cholangiocarcinoma. Liver Transpl.

28. Matthaei H, Krieg A, Schmelzle M, Boelke E, Poremba C, et al. (2009) Longterm survival after surgery for primary hepatic sarcoma in adults. Arch Surg 144: 339-344.

29. Alqahtani SA, Larson AM (2011) Adult liver transplantation in the USA. Curr Opin Gastroenterol 27: 240-247.

30. Husted TL, Neff G, Thomas MJ, Gross TG, Woodle ES, et al. (2006) Liver transplantation for primary or metastatic sarcoma to the liver. Am J Transplant 6: 392-397.

31. Maluf D, Cotterell A, Clark B, Stravitz T, Kauffman HM, et al. (2005) Hepatic angiosarcoma and liver transplantation: case report and literature review. Transplant Proc 37: 2195-2199.

32. Park H, Choi KH, Choi S-B, Choi JW, Kim DY, et al. (2011) Clinicopathological Characteristics in Combined Hepatocellular-Cholangiocarcinoma: A Single Center Study in Korea. Yonsei Med J 52: 753-760.

33. Yeh MM (2010) Pathology of combined hepatocellular-cholangiocarcinoma. J Gastroenterol Hepatol 25: 1485-1492. 
Citation: Nguyen NTT, Harring TR, Guiteau JJ, Cotton RT, de Armas IS, et al. (2011) Comparing Outcomes for Rare Primary Hepatic Tumors after Liver Transplantation. J Transplant Technol Res 1:106. doi:10.4172/2161-0991.1000106

34. Tang D, Nagano H, Nakamura M, Wada H, Marubashi S, et al. (2006) Clinical and pathological features of Allen's type $\mathrm{C}$ classification of resected combined hepatocellular and cholangiocarcinoma: a comparative study with hepatocellular carcinoma and cholangiocellular carcinoma. J Gastrointest Surg 10: $987-998$
35. Chan AC, Lo CM, Ng IO, Fan ST (2007) Liver transplantation for combined hepatocellular cholangiocarcinoma. Asian J Surg 30: 143-146.

36. Maganty K, Levi D, Moon J, Bejarano PA, Arosemena L, et al. (2010) Combined Hepatocellular Carcinoma and Intrahepatic Cholangiocarcinoma: Outcome After Liver Transplantation. Dig Dis Sc 55: 3597-3601. 